\title{
Mirtazapine-Induced Thrombocytopenia and Neutropenia
}

\section{Mirtazapin İlişkili Trombositopeni ve Nötropeni}

\author{
Selami K. Toprak ${ }^{1}$, Elçin Erdogan ${ }^{1}$, Özlem Kurt Azap ${ }^{2}$ \\ ${ }^{1}$ Baskent University, School of Medicine, Department of Hematology, Ankara, Turkey \\ ${ }^{2}$ Baskent University, School of Medicine, Department of Clinical Microbiology and Infectious Disease, Ankara, Turkey
}

\section{To the Editor,}

Neutropenia is a rare and commonly reversible side effect of antidepressant treatment [1]. Mirtazapine is a noradrenergic and specific serotonergic antidepressant that is approved for use in the treatment of major depression in many countries [2]. A major hematological side effect of mirtazapine is agranulocytosis. Herein we report a patient with depression that developed severe neutropenia-associated thrombocytopenia during treatment with mirtazapine; the patient was safely treated by switching to another drug.

A 72-year-old Caucasian male presented to the emergency room with fever and epistaxis. On presentation his white blood cell, neutrophil, and platelet counts were 3.27 $\times 10^{9} \mathrm{~L}^{-1}, 0.17 \times 10^{9} \mathrm{~L}^{-1}$, and $28.10 \times 10^{9} \mathrm{~L}^{-1}$, respectively. The patient was hospitalized with neutropenic fever and thrombocytopenia. Complete blood cell count from $20 \mathrm{~d}$ earlier was essentially normal.

The patient had a medical history of diabetes mellitus type 2 (regulated by diet), hypertension, and cervical spondylosis. His medication use included isosorbide5 -monohydrate and metoprolol succinate for 5 years. He had no obvious past history of hematological disorders. He did have a 1-month history of depressive symptoms, including low mood and alysosis. Because of his symptom profile $10 \mathrm{~d}$ earlier he was started on mirtazapine $15 \mathrm{mg}$ at night by the neurology department.

On physical examination he did not have organomegaly or lymphadenopathy; nor did he have ecchymoses or petechia/purpura. Laboratory findings, including immunoglobulin, vitamin B12, folate, and immunofixation levels were within normal limits. Other tests performed to determine a possible infectious etiology were negative. Peripheral blood smear showed thrombocytopenia and leucopenia/neutropenia. Bone marrow aspiration biopsy was performed with the permission of the patient. Bone marrow examination showed hypocellularity with differentiation of the myeloid lineage and mildly decreased megakaryocytes. There was no evidence of malign infiltration.

The absence of any other etiological mechanism, the patient's unremarkable laboratory work-up, and the close temporal association between administration of mirtazapine and the decrease in his neutrophil and thrombocyte counts strongly suggested that the bicytopenia was related to mirtazapine. As such, mirtazapine treatment was withdrawn due to the suspicion of its link to his symptoms. One week after discontinuation of mirtazapine escitalopram and alprazolam were initiated following psychiatric consultation due to his labile and bad affect. The patient's platelet count increased to $89.00 \times 10^{9} \mathrm{~L}^{-1}$, and then to $100.00 \times 10^{9} \mathrm{~L}^{-1}$ on the 7 th and 14th $\mathrm{d}$ of mirtazapine withdrawal, respectively. The absolute neutrophil and platelet counts increased to $2.5 \times 10^{9} \mathrm{~L}^{-1}$ and $219.00 \times 10^{9}$ $\mathrm{L}^{-1}$, respectively, $21 \mathrm{~d}$ after discontinuation of mirtazapine.

To the best of our knowledge like other tetracyclic antidepressants, mirtazapine is associated with severe neutropenia, but the mechanism of mirtazapine-associ- 
ated leucopenia (neutropenia) is not well understood [3]. Hypersensitivity and immune-mediated mechanisms have been suggested, including complement-mediated toxicity and drug-induced antibodies against committed stem cells, proliferating precursors, and mature blood cells [4]. Mirtazapine-induced thrombocytopenia is a rare hematological disorder; this serotonin reuptake inhibitor is not currently accepted as a definitive or probable cause of thrombocytopenia [5]. In a single case report that was published in 2003 Liu and Sahud researched the immunological nature of mirtazapine-induced thrombocytopenia [6]. The researchers identified mirtazapine-induced GPIIb/IIIa reactive antibodies in the patient's serum.

Tests to demonstrate drug-dependent antibodies were not performed in the presented patient and therefore the drug causality assessment was based on the relationship between drug intake and the onset of thrombocytopenia, as previously reported. Furthermore, the rapid increase in the patient's platelet count after discontinuation of mirtazapine therapy further indicated that neutropenia was due to mirtazapine treatment. Common causes of thrombocytopenia and leucopenia (neutropenia), including infections, were investigated using bone marrow aspiration biopsy and other laboratory investigations. Malignancy was excluded based on the clinical and histological pattern of thrombocytopenia/neutropenia.

To the best of our knowledge in vitro data show that platelet aggregation decreases when exposed to isosorbide, but thrombocytopenia and leucopenia (neutropenia) were not documented in clinical practice. Hematological side effects, including agranulocytosis, non-thrombocytopenic purpura and thrombocytopenic purpura have been associated with beta-blocking agents, in general. These side effects occur short-medium term after initiation of metoprolol therapy. In the presented case, metoprolol was used for 5 years and it was not stopped during the patient's hospitalization. These findings showed us that metoprolol and isosorbide were not the cause of thrombocytopenia/ neutropenia.

Neutropenia with cross intolerance between 2 tricyclics has been described, and there is evidence that patients can be successfully treated with another class of drug after such an incidence [1]. The absence of etiologic factors other than mirtazapine in the presented patient, such as different medications and disease, supported the hypothesis of mirtazapine-induced thrombocytopenia, suggesting an immune mechanism.

While collaboration with psychiatrists is important, clinicians (hematologist) should keep in mind that a primary care physician (psychiatrist) can fail to warn patients about thrombocytopenia/neutropenia or monitor thrombocyte and total-differential white blood cell counts in patients receiving mirtazapine at minimum several times during the first year of the therapy. As such, in patients treated with mirtazapine - especially older adults - clinicians should be aware that the drug can cause neutropenia/thrombocytopenia.

Written informed consent was obtained from the patient.

\section{Conflicts of Interest Statement}

None of the authors of this letter have any conflicts of interest, including specific financial interests, relationships, and/or affiliations, relevant to the subject matter or materials included.

\section{References}

1. Ozcanli T, Unsalver B, Ozdemir S, Ozmen M. Sertraline and mirtazapine-induced severe neutropenia. Am J Psychiatry 2005; 162 (7): 1386

2. Croom KF, Perry CM, Plosker GL. Mirtazapine: A review of its use in major depression and other psychiatric disorders. CNS Drugs 2009; 23 (5): 427-452

3. Davis J, Barkin RL. Clinical pharmacology of mirtazapine: Revisited. Am Fam Physician 1999; 60 (4): 1101

4. Dholakia R, Schleifer SJ, Ahmad YJ, Narang IS. Delayedonset mirtazapine-related leucopenia and rechallenge. J Clin Psychopharmacol 2010; 30 (6): 758

5. Reese JA, Li X, Hauben M, Aster RH, Bougie DW, Curtis BR, George JN, Vesely SK. Identifying drugs that cause acute thrombocytopenia: An analysis using 3 distinct methods. Blood 2010; 116 (12): 2127-2133

6. Liu X, Sahud MA. Glycoprotein IIb/IIla complex is the target in mirtazapine-induced immune thrombocytopenia. Blood Cell Mol Dis 2003; 30 (3): 241-245 